\title{
Wet Process Induced Phase Transited Drug Delivery System as a Means for Delivery of Gastrointestinal Irritant Drug: Histomorphological Analysis
}

\author{
A.K. PHILIP* AND K. PATHAK \\ Department of Pharmaceutics, Rajiv Academy for Pharmacy, Mathura-286001, Uttar Pradesh, \\ India.
}

\begin{abstract}
A non-disintegrating, asymmetric membrane capsular system for a poorly water soluble drug, flurbiprofen, was developed and evaluated in vitro and in vivo. Asymmetric membrane capsules were made by phase inversion. The effect of varying osmotic pressure of the dissolution medium on drug release was studied. Acute toxicity studies and histomorphological analysis were conducted in rats. Scanning electron microscopy showed an outer dense region with few pores and an inner porous region on the prepared asymmetric membrane. Statistical tests on in vitro release studies were applied at $p>0.05$. The drug release was found to be independent of the $\mathrm{pH}$ but dependent on the osmotic pressure of the dissolution medium. The results of in vivo toxicity studies may support the use of phase transited asymmetric membrane capsules as a means for delivery of gastro-intestinal irritant drugs in a controlled manner through Fickian diffusion.
\end{abstract}

Key words: Asymmetric membrane capsule, acute toxicity, histomorphological, intentional defect, osmotic pressure.

\section{INTRODUCTION}

There has been increasing interest in development of osmotic devices in the past two decades and various osmotic pumps have been reviewed [1]. The first device using osmotic principles to deliver active ingredients was reported in the 1950s [2]. The elementary osmotic pump (EOP) was first introduced in the 1970s [3]. In its operation, the osmotic core imbibes water from the surrounding medium via the semipermeable membrane. Subsequently, a drug solution is generated within the device and delivered out of the device via the delivery orifice [4]. However, this type of EOP was only suitable for the delivery of water soluble drugs. To overcome this limitation of the EOP, a pushpull osmotic tablet was developed. The pushpull osmotic tablet also suffered disadvantages [5]. To avoid sophisticated techniques in the design of osmotic tablet systems, the monolithic osmotic tablet system has been studied [6]. Osmotic tablets with an asymmetric membrane coating, which can achieve high water fluxes, have been described previously [7].

Asymmetric membrane capsules (AMC) prepared both by wet and dry processes are examples of a single core osmotic delivery system consisting of a drug containing core surrounded by an asymmetric membrane [89]. The in vitro and in vivo functionality of AMC have been reviewed by Philip and Pathak [10]. One of the advantages of asymmetric membrane is a higher rate of water influx, allowing release of drugs with a lower osmotic pressure or lower solubility [11]. Newer and more effective asymmetric membrane coatings have been developed for osmotic drug delivery [12]. In addition, dual coating AMCs have been successfully 
repared for delivery of poorly water soluble drugs [13].

Flurbiprofen is a useful nonsteroidal antiinflammatory drug, effectively used in the treatment of rheumatoid arthritis [14], osteoarthritis, mild to moderate pain [15] and ocular inflammatory conditions [16]. Because of its short elimination half-life $(4 \mathrm{~h})$, multiple dosing is required to achieve and maintain therapeutic concentrations. This, however, may result in adverse gastrointestinal (GI) reactions [17].

The objectives of the present study were to develop and evaluate wet process induced phase transited AMCs to deliver the poorly water soluble drug, flurbiprofen in a controlled manner; and to evaluate the ability of the fabricated phase transited dosage form in preventing gastric disturbances in rats.

\section{MATERIALS AND METHODS}

\section{Materials and Reagents}

Flurbiprofen was obtained from Sun Pharmaceuticals Pvt. Ltd (Gujarat, India). Sodium dihydrogen phosphate and disodium hydrogen phosphate (S. D. Fine Chemicals, Mumbai, India) were of analytical grade. Ethylcellulose (EC, $50 \mathrm{cps}$ ), acetone, glycerin and ethyl alcohol were procured from Qualigens Pvt. Ltd (Mumbai, India) while mannitol was purchased from C.N. Chemicals, (Uttar Pradesh, India). Solvents of reagent grade and double distilled water were used in all experiments.

Wistar albino rats were obtained from Rajiv Academy for Pharmacy, Mathura, India. The animal experiments were conducted after prior approval from the ethical committee of Rajiv Academy for Pharmacy, Mathura (IAEC/RAP/1376).

A Shimadzu 1700 UV/VIS spectrophoto- meter (Shimadzu Corp., Kyoto, Japan) was used to carry out UV spectrophotometric analysis. InStat software (Graphpad Software Inc, San Diego, CA, USA) was employed in statistical analysis. Photographs were taken using an Olympus 510 camera (Olympus, Tokyo, Japan) with Microimage process software (DA1-180 M v 2.01). A Kyowa medilux-12 microscope (Kyowa Optical Company Ltd, Tokyo, Japan) was employed in histomorphological studies.

\section{Preparation of Asymmetric Membrane Capsules}

The AMCs were prepared by using the wet phase inversion process. The membranes were precipitated on glass mold pins of diameter $5.52 \mathrm{~mm} \pm 0.05$ and $6.1 \mathrm{~mm} \pm 0.022$ for the body and cap, respectively. The glass mold pins were coated by dipping in a solution of 10 or $15 \%$ w/v EC and varying amounts of glycerol dissolved in acetone and ethanol as shown in table 1 . The pins were air dried for $15 \mathrm{~s}$ and immersed in an aqueous quenching solution containing $10 \% \mathrm{w} / \mathrm{v}$ of glycerol for $10 \mathrm{~min}$ in order to generate phase transited asymmetric membranes. Asymmetric membranes in the shape of the body and cap of conventional capsules were then stripped off after removal from the quench bath, dried at ambient temperature for at least $8 \mathrm{~h}$ and trimmed to fit inside each other to form the phase transited AMC.

About $200 \mathrm{mg}$ of the drug (mixed with or without $50 \mathrm{mg}$ of mannitol) was loaded after passing through a 100 mesh sieve. The AMCs were filled manually. Mannitol was used as an osmogen because flurbiprofen was found to be osmotically inactive [18]. The filled AMCs were sealed with an ethanolic solution of EC. The composition of all the AMCs formed is represented in Table 1. Citric acid was incorporated in AMC9 as solubility enhancer. 
Table 1. Composition of the 9 AMC formulations.

\begin{tabular}{|c|c|c|c|c|c|c|c|c|c|c|}
\hline & \multirow[b]{2}{*}{ Variable } & \multicolumn{9}{|c|}{ AMC } \\
\hline & & 1 & 2 & 3 & 4 & 5 & 6 & 7 & 8 & 9 \\
\hline 1 & Ethylcellulose (\% w/v) & 10 & 15 & 10 & 15 & 10 & 15 & 10 & 15 & 10 \\
\hline 2 & Mannitol (mg) & 0 & 0 & 50 & 50 & 0 & 0 & 50 & 50 & 50 \\
\hline 3 & Glycerol $(\% \mathrm{w} / \mathrm{v})$ & 8 & 8 & 8 & 8 & 20 & 20 & 20 & 20 & 8 \\
\hline 4 & Quenching concentration $(\% \mathrm{w} / \mathrm{v})$ & 10 & 10 & 10 & 10 & 10 & 10 & 10 & 10 & 10 \\
\hline 5 & Quenching time (minutes) & 10 & 10 & 10 & 10 & 10 & 10 & 10 & 10 & 1 \\
\hline 6 & Acetone $(\% \mathrm{v} / \mathrm{v})$ & 50 & 50 & 50 & 50 & 50 & 50 & 50 & 50 & 50 \\
\hline 7 & Ethanol $(95 \%)(\% \mathrm{v} / \mathrm{v})$ & 30 & 30 & 30 & 30 & 25 & 25 & 25 & 25 & 30 \\
\hline 8 & Water $(\mathrm{mL})$ & 90 & 90 & 90 & 90 & 90 & 90 & 90 & 90 & 90 \\
\hline 9 & Citric acid (mg) & 0 & 0 & 0 & 0 & 0 & 0 & 0 & 0 & 25 \\
\hline
\end{tabular}

\section{Scanning Electron Microscopy (SEM)}

The AMCs obtained were examined for their porous structure using Jeol 6100 SEM (Jeol, Tokyo, Japan) before and after complete dissolution of the core contents. After dissolution, the membranes were dried at $50{ }^{\circ} \mathrm{C}$ for $8 \mathrm{~h}$ and stored in a dessicator before examination. The asymmetric membrane surfaces were sputter-coated for 5 to $10 \mathrm{~min}$ with gold using a fine coat ion sputter and examined under SEM.

\section{In vitro drug release}

In vitro cumulative drug release from prepared formulations $(n=6)$ were studied using British Pharmacopoeia type 2 apparatus with paddle rotation speed of $75 \mathrm{rpm}$ at a temperature of $37 \pm 0.5^{\circ} \mathrm{C}$. The dissolution medium was $900 \mathrm{ml} 0.1 \mathrm{~N} \mathrm{HCl}$ (simulated gastric fluid) $\mathrm{pH} 1.2$ for the first $2 \mathrm{~h}$, followed by $900 \mathrm{ml}$ phosphate buffer (simulated

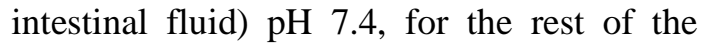
experiment. One $\mathrm{ml}$ of sample was withdrawn at specified time intervals, diluted with fresh dissolution medium and subjected to UV spectrophotometric analysis at $247 \mathrm{~nm}$.

\section{Statistical analysis}

The release profiles up to $t_{50 \%}$ of flurbiprofen from all the formulations $(n=6)$ in the dissolution medium were statistically compared with the release profiles of the marketed formulation using Dunnett multiple comparison $\mathrm{t}$ test. The statistical significance was tested at $\mathrm{p}>0.05$. The best formulation was chosen after pair wise comparison using the dissimilarity factor $\left(f_{1}\right)$. The formulation with the lowest $f_{1}$ value in the Fickian diffusion release mechanism was considered the best.

\section{Effect of varying osmotic pressures of the dissolution medium}

Release studies of the optimized formulation were conducted in media of different osmotic pressures in order to determine the mechanism of flurbiprofen release. Simulated intestinal fluid was selected as the dissolution medium for this study because the drug has good solubility in this medium. To increase the osmotic pressure of the medium, mannitol was added and the $\mathrm{pH}$ adjusted to $7.4 \pm 0.5$.

Different osmotic pressures in the dissolution medium were created by incorporating mannitol in a series of increasing concentration namely $10 \mathrm{mg}$ (AMC 3a), 20 $\mathrm{mg}$ (AMC 3b), $30 \mathrm{mg}$ (AMC 3c), and $40 \mathrm{mg}$ (AMC 3d) to the amount already present within the formulation so as to create a decreasing osmotic gradient. 


\section{Kinetics of drug release}

In general, release of drug from an osmotic system depends upon several factors including osmotic pressure, pore size and coating thickness. In vitro release from the marketed formulation $(50 \mathrm{mg}$, without polymer) exhibited fast release, with over $80 \%$ released in the first hour. Drug release from the formulations containing polymer was more controlled, with $t_{50 \%}$ achieved in more than $10 \mathrm{~h}$. In order to describe kinetics of drug release from the drug delivery systems, various mathematical equations have been proposed namely zero order [19], first order [20], Higuchi model [21] and Hixson-Crowell cube root models [22]. In order to authenticate the release model, dissolution data can further be analyzed by the Korsymeyer-Peppas equation [23].

\section{In vivo study conditions}

Healthy male and female Wistar albino rats aged 2-3 months and weighing 250-300 g were selected for the study. All the rats were housed individually in polypropylene cages and subjected to a $12 \mathrm{~h}$ light/dark cycle, 25$30{ }^{\circ} \mathrm{C}$ and $35-60 \%$ humidity in the animal house. The animal experiments were carried out in accordance with internationally accepted ethical guidelines for the care of laboratory animals. Prior to the experiments, the rats were allowed to adapt to the laboratory conditions for one week. They were maintained on standard diet before and during the study. Sixteen hours before the experiments, they were fasted overnight but allowed free access to water. Eighteen rats were recruited for the study, divided into 3 groups of 3 males and 3 females each and treated as follows:

Group 1: Control animals for acute toxicity studies of flurbiprofen, not subjected to experimentation.

Group 2: Reference animals for acute toxicity studies, treated with pure flurbiprofen.

Group 3: Test animals for acute toxicity studies, treated with test formulation containing flurbiprofen.

\section{Acute toxicity studies}

Healthy adult Wistar rats of either sex, starved overnight, were subjected to acute toxicity studies as per the revised ICH guidelines (61 FR 43934) to determine the superiority of the specialized dosage form. The reference rats were given an oral suspension of the pure drug in demineralized water while the test animals were administered with the dosage form. The pure drug formulation and the test formulation were given at a dose toxic to rats $(117 \mathrm{mg} / \mathrm{kg})$ by use of a suitably designed stainless steel rod. Male and female rats were identified bya tail mark and kept in separate standard cages. They were fed $3 \mathrm{~h}$ after dosing and daily on standard diet for 14 days. The study animals were observed for mortality and signs of intoxication at $0.5,1,2,4$ and $6 \mathrm{~h}$ following dosing and thereafter twice a day for 14 days. The weights of the rats were individually recorded before and after dosing for 14 days. Gross necropsies were performed on all animals including those sacrificed, moribund, found dead or terminated at 14 days. All the specimens were subjected to photographic impression and histopathological studies at termination.

\section{Photographic impressions of the stomach}

To observe for any untoward changes after administration of the dosage forms, three different sets of photographic images were taken, one each for the control, reference and test animals.

\section{Histomorphological analysis}

Histomorphological analysis of the gastric mucosa of the rats was conducted to evaluate any pathological changes occurring in the cell morphology and tissue organization. The epithelial tissues were fixed in a $10 \%$ neutral 
buffered formalin solution for $1 \mathrm{~h}$, washed in water for $30 \mathrm{~min}$, dehydrated using $95 \%$ ethanol and embedded in paraffin using standard procedures. The tissue samples were then cut into $5 \mu \mathrm{m}$ thick sections using a microtome and conventionally stained using hematoxylin-eosin. Three slides of blank control, reference slide and test slide were prepared. Photomicrographs of the slides were taken at magnification $15 x, 5 x$ and $10 x$.

\section{RESULTS AND DISCUSSION}

\section{Scanning Electron Microscopy (SEM)}

For the SEM studies, $15 \%$ w/v EC membranes with varying proportions of pore forming agent (glycerol) were obtained before and after complete dissolution. The SEM revealed that the membrane obtained before dissolution ( $8 \% \mathrm{w} / \mathrm{v}$ glycerol) had an outer dense nonporous region and an inner lighter porous region (Figure $1 \mathrm{~A}$ ). After complete dissolution, the exhausted membrane showed a large number of pores in a net-like structure (Figure $1 \mathrm{C}$ ). The formulation prepared with this membrane did not show swelling or rupturing. The membrane containing $20 \%$ w/v of glycerol showed similar nonporous but larger inner porous regions (Figure 1 B) with swelling or elongation but no rupture at the end the dissolution study while that containing a higher proportion of glycerol $(25 \% \mathrm{w} / \mathrm{v})$ showed larger pores and caused bursting within an hour of the dissolution study. Thus the SEM studies showed that the concentration of plasticizer is an important parameter in deciding membrane strength with high concentrations of glycerol making the asymmetric membrane correspondingly weak eventually causing rupture.

\section{In vitro drug release studies}

In vitro studies were performed for the experimental and market formulations. The results showed that incorporation of mannitol (AMC 3) resulted in development of significant osmotic pressure inside the capsular system, which increased the release rate of flurbiprofen (Figure 2).

This effect was also evident during the study of the individual effect of mannitol which showed a decrease of $49.07 \mathrm{~min}$ in the attainment of $\mathrm{t}_{50 \%}$ from AMC 3. Higher concentrations of the pore former (glycerol) enhanced the release from this formulation probably due to increased pore formation on the membrane during dissolution, thus causing burst release. High EC concentration constrained the release of flurbiprofen from

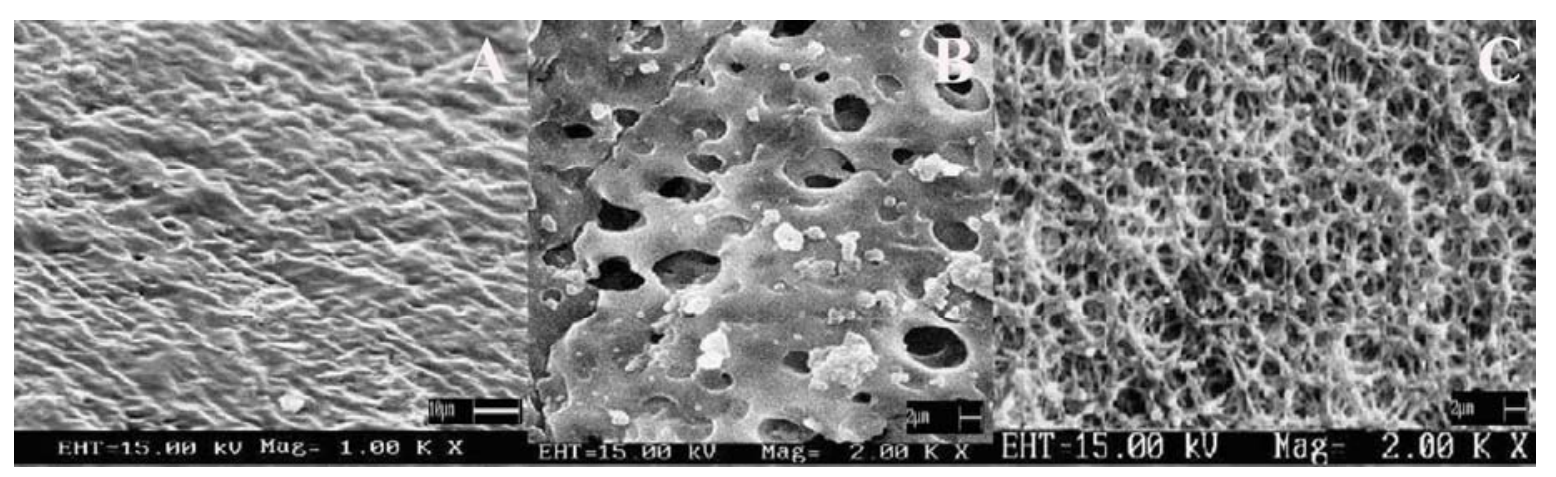

Figure 1. SEM of coating membrane obtained: A) Before dissolution, showing outer dense nonporous region and containing $8 \%$ w/v glycerol at $1000 \times$; B) Before dissolution showing large inner porous region and containing $20 \% \mathrm{w} / \mathrm{v}$ glycerol at $2000 \times$; C) After complete dissolution showing net-like structure and containing $25 \%$ w/v glycerol at $2000 \times$. 
the capsular membrane as compared with formulation AMC 1 probably due to increased diffusional path for the drug during its release into the dissolution medium. The individual effect of high EC concentration induced an increase of $87.22 \mathrm{~min}$. Interactive studies showed a delayed achievement of $\mathrm{t}_{50 \%}$ in AMC 8.

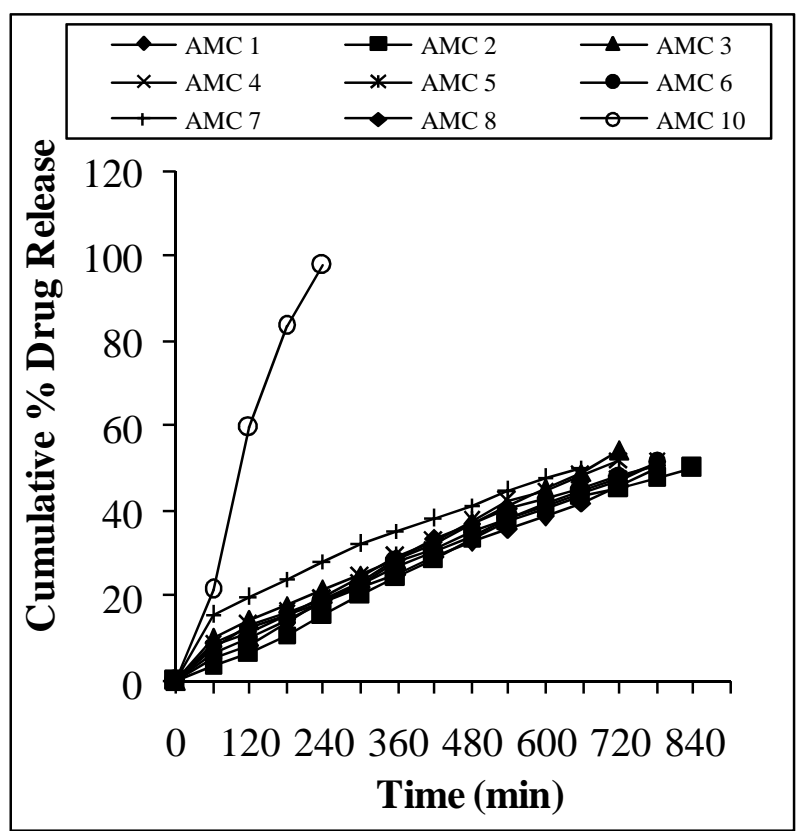

Figure 2. Comparative in vitro dissolution profiles for formulations AMC 1-AMC 8 along with the marketed formulation (AMC 10).

A systematic study of the interaction between the 3 variables showed that there was an increase of $9.03 \mathrm{~min}$. This may have arisen as a result of increased drug holding capacity of the polymer at a higher EC concentration coupled with the swelling of the asymmetric membrane. This suggests that the membrane thickness has a prominent role in constraining the release of flurbiprofen.

However, this delay was greatly reduced by the burst release of flurbiprofen resulting from individual effects of the other 2 variables. The marketed formulation (AMC 10) showed an initial burst release which was only comparable to the best formulation with solubility enhancer for the drug (AMC 9) [8].

\section{Statistical analysis}

Statistical analysis was performed using Dunnett's Multiple Comparison Test. The dissimilarity factor $\left(f_{1}\right)$ given in scale-up and postapproval changes (SUPAC) guidelines [24] for modified dosage forms was used to further justify the selection of the best formulation which showed least significance during multiple t-test at $\mathrm{p}>0.05$. Dunnett's Multiple Comparison Test compared all the formulation with the marketed formulation of flurbiprofen (AMC 10). Values were calculated for all the formulations $(\mathrm{q}<1.641$, $\mathrm{F}=0.2567$ and $\mathrm{p}=0.9476$ ). The test was run at $95 \%$ confidence level and the differences between all formulations as compared to the marketed formulation were found to be statistically insignificant. The $\mathrm{q}$ value for AMC 3 was the least (0.0345) among all the formulations. Dissimilarity factor $\left(f_{1}\right)$ with respect to Fickian diffusion between AMC 3 and AMC 10 was found to be 6.12, suggesting that the two formulations have completely different dissolution profiles.

\section{Effect of varying osmotic pressure}

Since the study was based on osmotic delivery, the effect of varying osmotic pressure on release profiles of the optimized formulation, AMC 3 was studied (Figure 3). An $r^{2}$ value of 0.9868 was obtained for the plot of initial release rate against external osmotic pressure difference, thus implying that osmotic pumping was a primary mechanism governing drug release from the developed formulations.

\section{Kinetics of drug release}

Release models were applied on all the formulations (AMC 1 to AMC 9). The results showed that the data for AMC 2 to AMC fits zero order, first order, Higuchi, Hixon Crowell model and Korsmeyer- 
Peppas models. While considering the higher correlation coefficient value (r), the release data fits zero order model better. However, on basis of the highest correlation coefficient value $(r)$ of release models, AMC 3 and AMC 9 were the best formulations. Drug release mechanism, using drug release data for AMC 3 and AMC 9 formulations was used to further analyze for curve fitting based on the Korsmeyer-Peppas model. AMC 3 gave the parameters $\mathrm{n}=0.4617, \mathrm{k}=0.6369$ and $\mathrm{r}=$ 0.9998 while AMC 9 showed $\mathrm{n}=0.4053$, $\mathrm{k}=3.6749$ and $\mathrm{r}=0.9783$. This was confirmatory that release of flurbiprofen from AMC 3 and AMC 9 formulation was via Fickian diffusion.

\section{Photographic impressions of the stomach}

Acute toxicity studies as per the revised guidelines of International Conference on Harmonization were conducted and photographic impressions taken to investigate

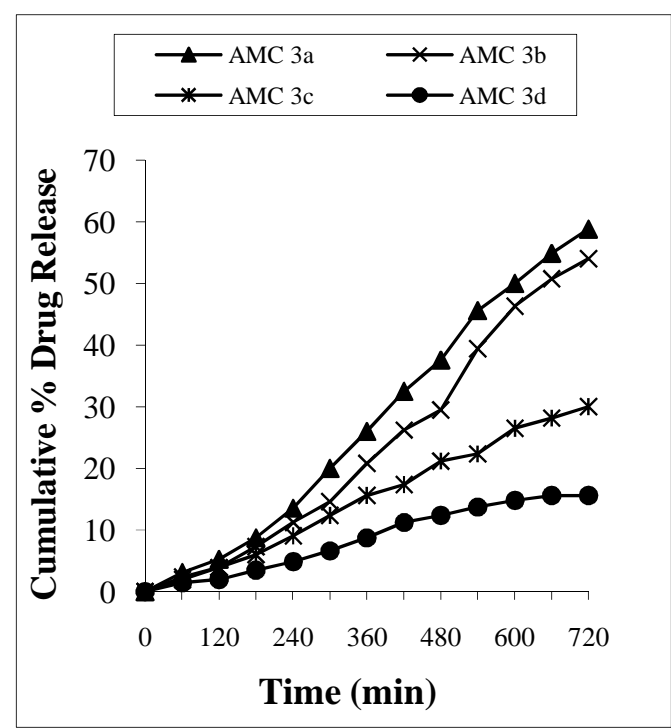

Figure 3. Comparison of in vitro KTP release From AMC 3 in dissolution media of different osmotic pressures. AMC 3a-3d contained an additional 10, 20, 30 and $40 \mathrm{mg}$, respectively, of mannitol.

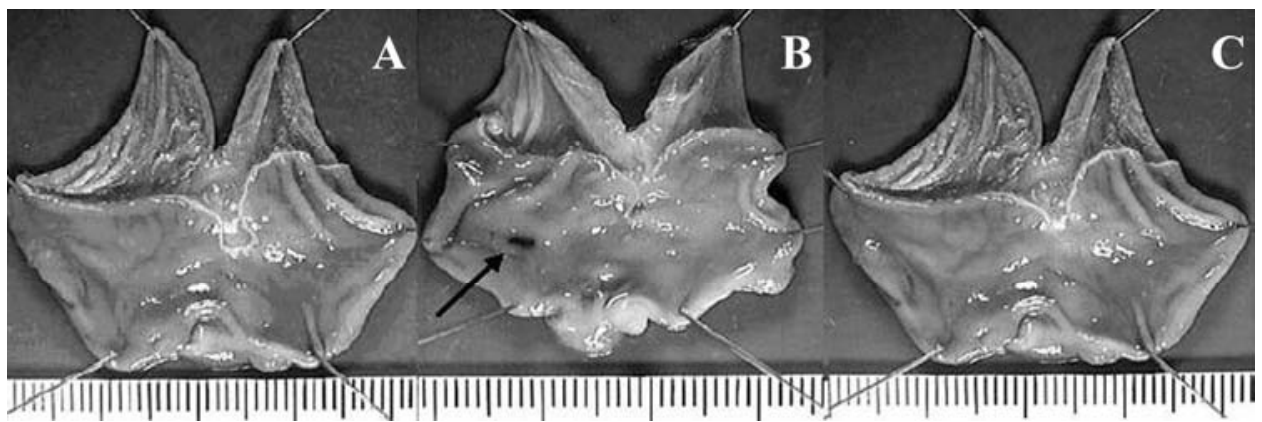

Figure 5. Photographs of inside of stomach: A) Control group for flurbiprofen; B) Perforation visible when pure flurbiprofen was administered C) No perforations visible after phase transited dosage form of flurbiprofen was administered.

the safety of the prepared formulation as compared to administration of pure drug. Photographs taken of the inner stomach wall (Figure 5 A) clearly showed a marked difference between administration of the pure drug (Figure $5 \mathrm{~B}$ ) and the prepared dosage form (Figure $5 \mathrm{C}$ ). This finding may probably be due to the fact that when the pure drug was administered, poor aqueous solubility in the gastric medium resulted in the gastric side effects usually associated with the drug. Conversely, the synthesized asymmetric membrane has a non-disintegrating polymeric capsular system which allows only the dissolved amount of drug to come out into the simulated gastric fluid from the capsular system. Undissolved drug is thereby prevented from coming into contact with the gastric mucosa. 


\section{Histomorphological analysis}

Histomorphological analysis of the gastric mucosa (Figure $6 \mathrm{~A}$ ) using photomicrograph showed results similar to the photographic impressions of the inside of the stomach wall. Disturbance of the gastric mucosa were seen when the drug was administered in its pure form (Figure $6 \mathrm{~B}$ ) as opposed to the synthesized dosage form (Figure $6 \mathrm{C}$ ). The histomorphological profile confirms the safety of the drug for oral administration with the synthesized dosage form.

\section{CONCLUSION}

Phase transited asymmetric membrane polymeric capsules were developed not only for controlled delivery of flurbiprofen but also reducing undue gastric disturbance caused by the drug. The release of flurbiprofen was shown to be independent of $\mathrm{pH}$ and intentional defect on the release membrane but dependant on osmotic pressure of the dissolution medium.

\section{ACKNOWLEDGEMENT}

The authors are indebted to Sun Pharmaceuticals for generously supplying a free gift sample of flurbiprofen and IITR, Roorkee, India for their support in obtaining SEM photographs.

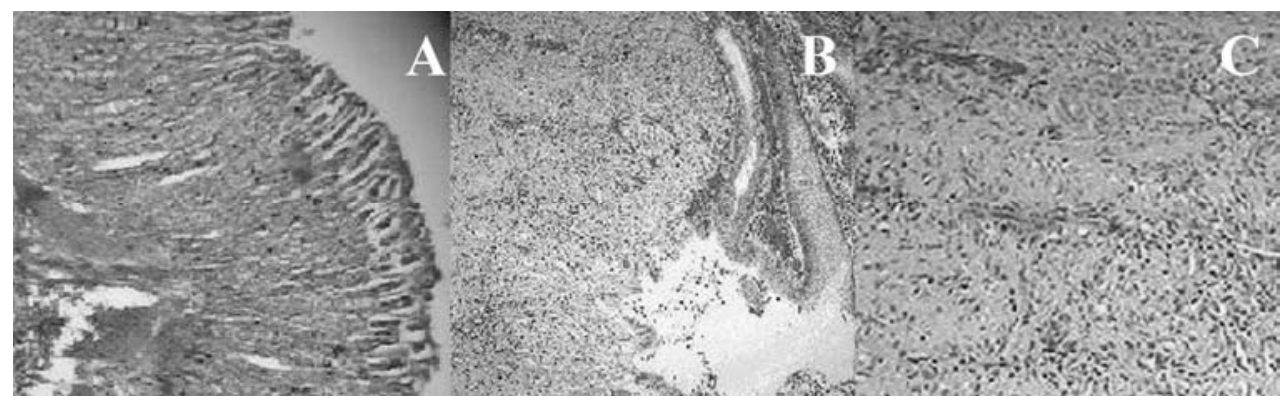

Figure 6. Histopathologic conditions of the gastric mucosa: A) Control group for flurbiprofen at $15 \times 3)$ Disturbance of gastric mucosa visible when pure flurbiprofen is administered at $5 \times$ C) No disturbance visible when phase transited dosage form of flurbiprofen administered at $10 \times$.

\section{REFERENCES}

[1] G. Santus and R.W. Baker, J. Control. Rel. 35 (1995) 1-21.

[2] S. Rose and J.F. Nelson, Aust. J. Exp. Bio. 33 (1955) 410-415.

[3] F. Theeuwes, J. Pharm. Sci. 64 (1975)1987-1991.

[4] L. Liu, J. Ku, G. Khang, B. Lee, J.M. Rhee and H.B. Lee, J. Control. Re. 68 (2000) 146-54.
[5] F. Theeuwes F, R.J. Saunders and W.S. Mefford, U.S. Patent 4,088,864 (1978).

E.X. Lu, Z.Q. Jiang, Q.Z. Zhang and X.G. Jiang, J. Control. Rel. 92 (2003) 375-382.

[7] S.M. Herbig, J.R. Cardinal, R.W. Korsmeyer and K.L. Smith, J. Control. Rel. 35 (1995) 127-136. 
[8] A.K. Philip and K. Pathak, AAPS Pharm. Sci. Tech. 7 (2006) E1-E11.

[9] A. Philip and K. Pathak, PDA J. Pharm. Sci. Tech. 61 (2007) 24-36.

[10] A.K. Philip and K. Pathak, Pharma Times 39 (2007) 24-25.

[11] C.H. Wang, H.O. Ho, L.H. Lin, Y.K. Lin and M.T. Sheu, Int. J. Pharm. 297 (2005) 89-97.

[12] Y.K. Lin and H.O. Ho, J. Control. Rel. 89 (2003) 57-69.

[13] A.K. Philip, K. Pathak and P. Shakya, Eur. J. Pharm. Biopharm. (Accepted) doi:10.1016/j.ejpb.2007.12.011.

[14] C.C. Marsh, A.A. Schuna and W.R. Sundstorm, Pharmacotherapy 6 (1986) 10-16.

[15] A.G. Gilman, T.W. Rall and P. Taylor, Goodman and Gillman's The Pharmacological Basis of Therapeutics, Volume 11. Pergamon Press, New York. 1990, p 1632.
[16] V.T. Thaller, M.K. Kulshrestha and K. Bell, Eye 14 (2000) 642-645.

[17] C. Chandran, A. Roy and R.N. Saha,Ind. J. Pharm. Educ. 39 (2005) 22-26.

[18] Martin, Physical Pharmacy, Vol. 4. B.I. Waverly Pvt Ltd, India. 1999, p 74.

[19] N. Najib and M. Suleiman, Drug Dev. Ind. Pharm. 11 (1985) 21692181.

[20] S.J. Desai, P. Singh, A.P. Simonelli and W.I. Higuchi, J. Pharm. Sci. 55 (1966) 1230-1234.

[21] T. Higuchi, J. Pharm. Sci. 52 (1963) 1145-1149.

[22] A.W. Hixson and J.H. Crowell, Ind. Eng. Chem. 23 (1931) 923-931.

[23] R.W. Korsmeyer, R. Gurny, E.M. Doelker, P. Buri and N.A. Peppas, Int. J. Pharm. 15 (1983) 25-35.

[24] J.B. Dressman, H. Lennernas, Oral Drug Absorption, Vol. 106. Marcel Dekker Inc., New York. 2000, p 183. 